\title{
Development of super-resolution optical inspection system for semiconductor defects using standing wave illumination shift
}

\author{
S. Usuki*, H. Nishioka, S. Takahashi and K. Takamasu \\ Department of Precision Engineering, The University of Tokyo \\ Hongo 7-3-1, Bunkyo-ku Tokyo 113-8656 JAPAN
}

\begin{abstract}
Semiconductor design rules and process windows continue to shrink, so we face many challenges in developing new processes such as $300 \mathrm{~mm}$ wafer, copper line and low-k dielectrics. The challenges have become more difficult because we must solve problems on patterned and un-patterned wafers. The problems include physical defects, electrical defects, and even macro defects, which can ruin an entire wafer rather than just a die. The optics and electron beam have been mainly used for detecting of the critical defects, but both technologies have disadvantages. The optical inspection is generally not enough sensitive for defects at $100 \mathrm{~nm}$ geometries and below, while the SEM inspection has low throughput because it takes long time in preparing a vacuum and scanning $300 \mathrm{~mm}$. In order to find a solution to these problems, we propose the novel optical inspecting method for the critical defects on the semiconductor wafer. It is expected that the inspection system's resolution exceed the Rayleigh limit by the method. Additionally the method is optical one, so we can expect to develop high throughput inspection system. In the research, we developed the experimental equipment for the super-resolution optical inspection system. The system includes standing wave illumination shift with the piezoelectric actuator, dark-field imaging and super-resolution-post-processing of images. And then, as the fundamental verification of the super-resolution method, we performed basic experiments for scattered light detection from standard particles.
\end{abstract}

KEYWORDS: Optical inspection, Semiconductor defects, Standing wave illumination, Super-resolution

\section{INTRODUCTION}

The mass production of the 90nm-sized devices was begun in 2004, and the miniaturization of semiconductor devices has been accelerating and has reached the $65-\mathrm{nm}$ level. According to the international technology roadmap for semiconductors 2004, control of high-aspect ratio technologies such as damascene challenges all metrology methods [1]. In the roadmap, it is described the semiconductor defects inspection system for next-generation is urgently demanded, and requirement for sub-100-nm nodes and difficulty of defect detection challenge increase exponentially with shrinking design rules. Actually fabs are increasingly aiming to improve their products' reliability by introducing high-spec inspection system. One of the most important key-requirements is the defect detection of semiconductor wafer [2]. Defects in the wafer inspection field include random defect like killer particles, clustered defect, scratch defect and so on. These defects deteriorate electrical chip performance and process yield in factory line. Defect inspection also includes defect classification (categorizing) [3], defect size metrology and defect area identification in order to eliminate nuisance defects. Especially, the patterned wafer appearance inspection is one of the key-requirements for the next generation.

Optical method and electron beam are conventionally used for the semiconductor wafer inspection [4-5]. However inability of the optical inspection with the continuous miniaturization of interconnects has become the big issue. Electron beam is not useful for wider wafer inspection because it has low throughput property. We focused attention on the optical inspection because it has non-destructiveness, high throughput and potential for high resolution for sub-100nm inspection. In optical wafer inspection, resolution and defect detection beyond the Rayleigh limit that ordinary light can resolve are now required due to accelerating pattern miniaturization and development of advanced semiconductor devices [6].

*us@nano.pe.u-tokyo.ac.jp; phone and fax: +81-3-5841-6472

637508, (2006) - 0277-786X/06/\$15 - doi: 10.1117/12.690582 
One of the Solutions to challenges in semiconductor optical inspection is the use of a shorter wavelength, which has been studied as countermeasures against device miniaturization. However, the shortening of wavelength cannot keep up with the challenges and has a limit, so we propose the novel super-resolution inspection technique. When a pattern is miniaturized and made dense, light reflected from the wafer becomes weak, and the captured image becomes dark with low contrast, so we must develop high sensitive method and it must be able to obtain a lot of optical information [7-9]. Our super-resolution inspection technique combines standing wave illumination shift method with dark-field imaging technology to deliver optimal sensitivity for critical defect detection at sub-100-nm nodes and beyond without compromising on throughput. Standing wave illumination shift method enables the inspection optics to resolve patterns that the conventional method cannot. Nano-scale shift of illumination and the super-resolution post-processing are keys to achieving the resolution enhancement and higher sensitivity for the defect detection. The benefits of the superresolution inspection using standing wave illumination shift are the high resolution to critical defect on even the most challenging levels for $65-\mathrm{nm}$ design rules, and high performance at throughput at required sensitivity on $300 \mathrm{~mm}$ wafer.

\section{METHODOLOGY}

We describe principles of super-resolution using standing wave shift in this section. The system of the method is composed of standing wave illumination shift, scattering light modulation and super-resolution post-processing.

\subsection{Standing wave illumination shift and scattered light modulation}

We show the schematic explanation of the standing wave illumination shift and the scattered light modulation in figure 1. The standing wave illumination is generated by 2-beam interference with an objective lens. The standing wave illumination is scattered by the sample surface, and the scattered light is focused on the CCD imaging surface through the imaging lens. The standing wave is shifted at nano-scale by phase difference between 2-beam in the illumination (figure 1(a)). And then, the scattered light is modulated by the shift of the standing wave illumination (figure 1(b)). The super-resolution image can be calculated from multiple images by the super-resolution algorithm.

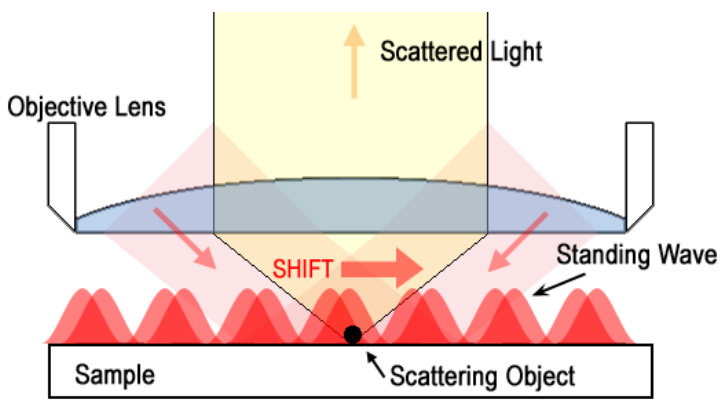

(a)

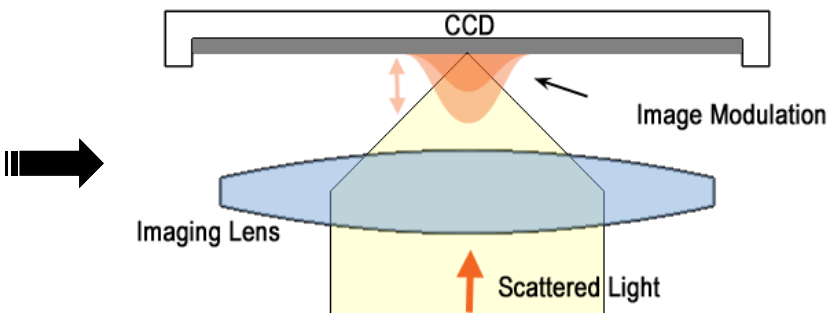

(b)

Figure 1. The schematic explanation of the standing wave illumination shift and the scattered light modulation. (a): The standing wave illumination shift and the scattered light from the scattering object. (b): The scattered light modulated by the shift of the standing wave illumination.

\subsection{Super-resolution algorithm for post-processing}

We show the schematic diagram of the super-resolution post-processing in the figure 2 . The super-resolution algorithm is as follows. Firstly, we define the illumination intensity $I_{i}$ shown in figure 3(a), and in this figure the number $i$ is the lateral position in the microscopic field. And then, the scattering efficiency $\alpha_{i}$ shown in figure 3(b) is defined. The scattering efficiency is an optical value, and it is a transformational ratio from the illumination intensity to scattering intensity. As we define the scattering intensity $S_{i}$ shown in figure 4, the relation among them is given as

$$
S_{i}=\alpha_{i} \times I_{i}
$$

In the imaging of the point source, it is needed to consider the diffraction, so we introduce diffraction contributing ratio $D$. This ratio is shown in figure 5, and the light detected with each pixel of CCD camera is a part of the light amount of the airy disk. In the figure 5, the scattering light of which intensity is $S_{i}$ is detected with CCD pixel at the position $j$ in the imaging field, and its light amount is $S_{i} \times D(|j-i|)$.

Then, the total light amount $X_{j}$ detected with the CCD pixel is given as 


$$
X_{j}=\sum_{i} s_{i} \times D(|j-i|)=\sum_{i} \alpha_{i} \times I_{i} \times D(|j-i|)
$$

, in which $s_{i}$ is scattering intensity at position $i$ and $D(|j-i|)$ is the diffraction contributing ratio at the position $j$. The $X$ (the imaging light amount obtained by CCD), $I$ (the illumination intensity determined by the generated standing wave) and $D$ (the diffraction contributing ratio determined by the optics) are known values, so $\alpha$ (the scattering efficiency) can be obtained by the formula (2) with the successive approximation. In this approximation, we set the initial value to constant value, and calculated iterative operation until convergence. To obtain $\alpha_{i}$ established within smaller area than the Rayleigh limit is to realize the super-resolution. Figure 6 shows an example of super-resolution imaging, which was numerically calculated by the super-resolution algorithm. From this theoretical analysis, we suggested the capability for resolving the 2-point scattering objects, which is not resolved with a conventional optical microscopy.

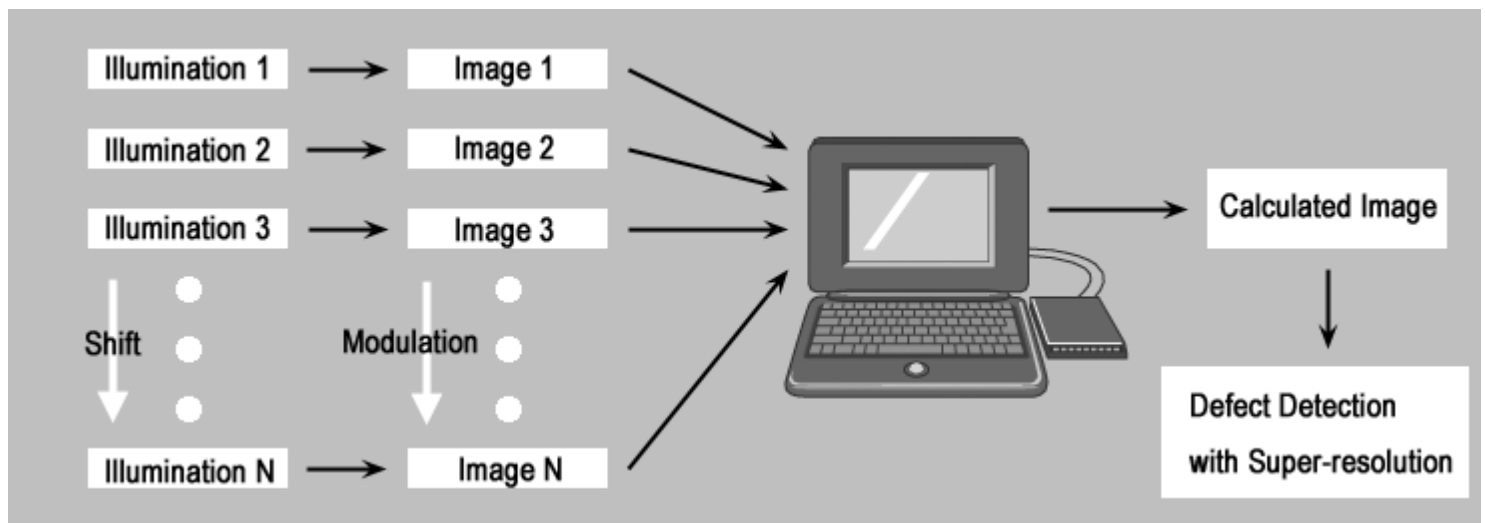

Figure 2. Image of sample resolved with super-resolution method is calculated from image 1 to $\mathrm{N}$ at the shifted positions of the standing wave illumination 1 to $\mathrm{N}$

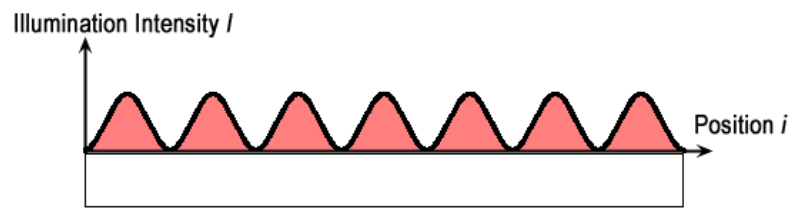

(a)

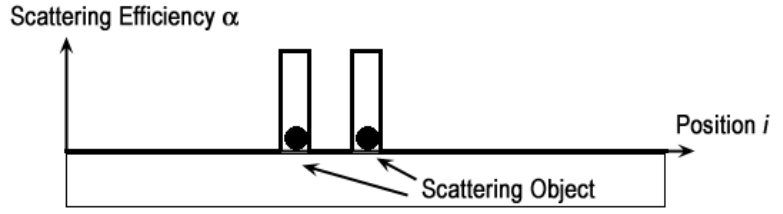

(b)

Figure 3. (a): Illumination intensity $I_{i}$; the number $i$ is the lateral position in microscopic field. (b): Scattering efficiency is an optical value, and it is a transformational ratio from the illumination intensity to scattering intensity

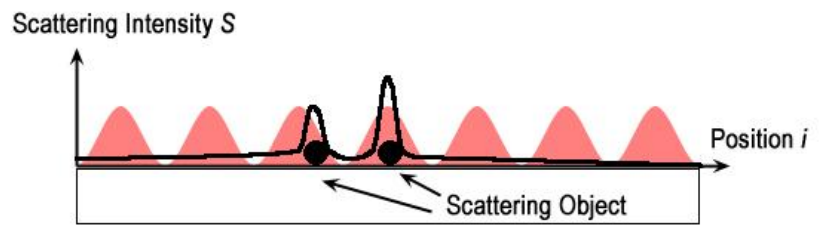

Figure 4. Scattering intensity is the product value of the illumination intensity and the scattering efficiency 


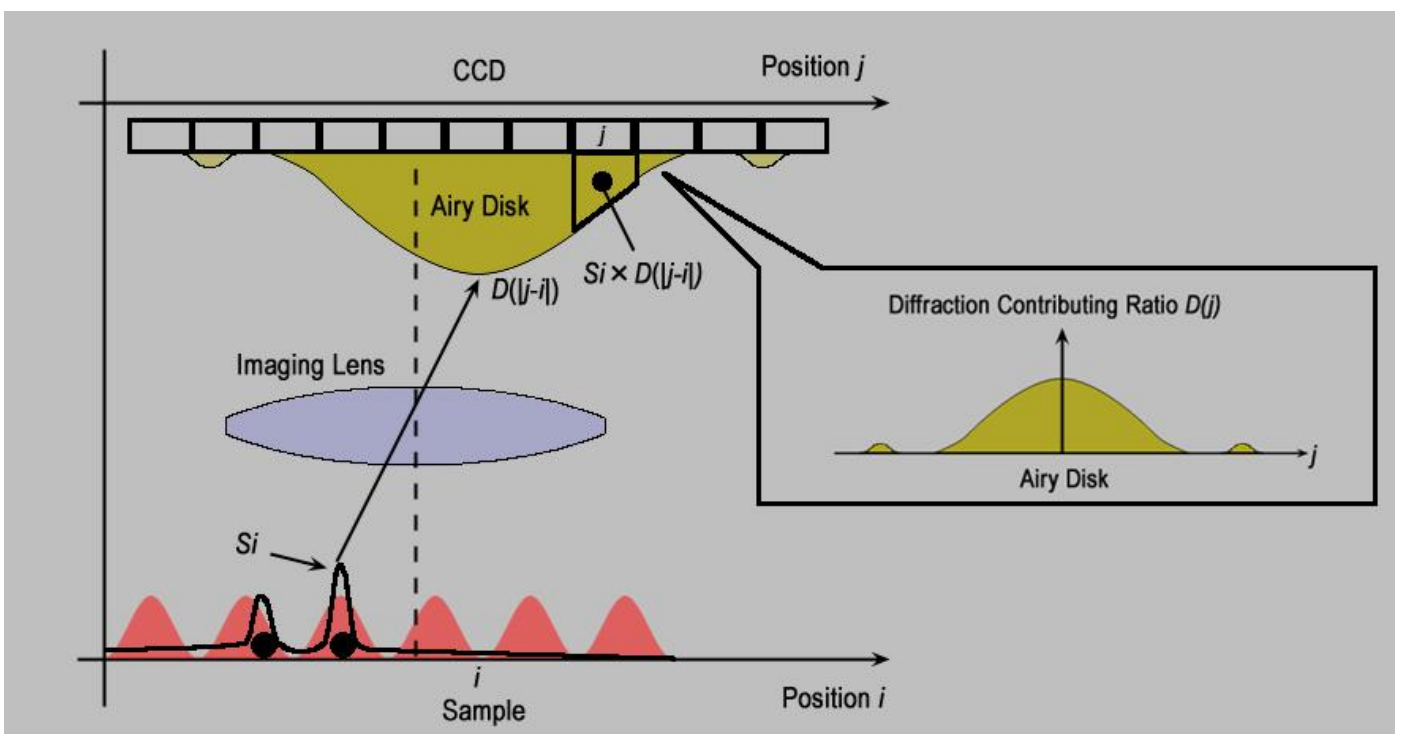

Figure 5. Relation among the scattering intensity $S_{i}$, the diffraction contributing ratio $D(|j-I|)$ and the light amount $X_{j}$. The diffraction contributing ratio $D$ is given by the airy disk function.

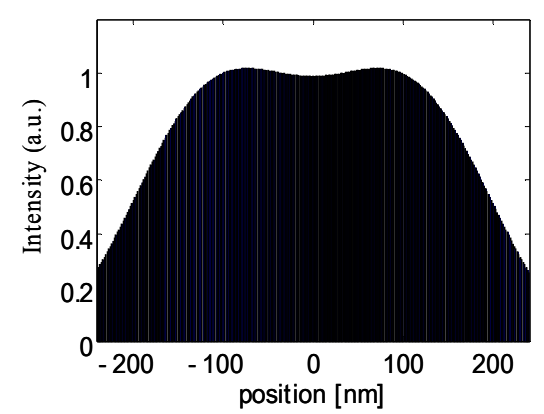

(a)

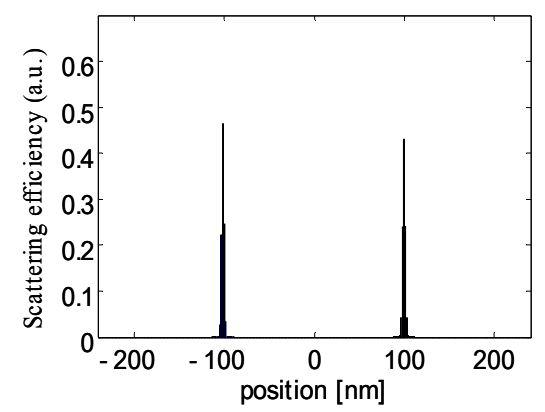

(b)

Figure 6. (a): 200nm-gapped 2-point object image with conventional method, (b): post-processed image of the 2-point using superresolution algorithm

\section{SUPER-RESOLUTION OPTICAL INSPECTION SYSTEM}

In order to verify the super-resolution method experimentally, we developed the experimental equipment for the standing wave illumination, the nano-shift of the standing wave and the dark-field imaging of the scattered light. Schematic diagram of the system of the super-resolution optical inspection using standing wave illumination shift is shown in figure 7.

In the figure 7, the beam splitter 1 divides a beam of light into 2 orthogonal beams. The 2 beams are collimated and facing in the microscopic field of the high power objective lens with high numerical aperture thorough mirror 1 , mirror 2 , mirror 3, mirror 4 , lens 1 , lens 2 , lens 3 , lens 4 and beam splitter 2 . The 2 collimated and facing beams interfere on the sample surface and generate standing wave illumination. The light axial position of the mirror 1 is displaced at nanoscale with the piezo-electric actuator, which produces phase difference between 2-beam in the illumination optics. Thus, the standing wave illumination can be shifted at nano-scale with the piezo-electric actuator attached to the mirror 1.

The standing wave illumination is scattered by the sample surface, and the scattered light is focused on the CCD imaging surface through the objective lens, beam splitter 2, lens 5, filter and tube lens. In the imaging optics, the objective lens and the lens 5 are set for the infinity correction optics, and the tube lens is set for the finite optics. The filter placed between the lens 5 and the tube lens, rejects the light that is the regular reflection of the illumination light. So we can 
obtain the only light from scattering factor on the sample surface with high contrast. This dark-field imaging of laser scattering makes it possible to detect the image with optically high signal-noise ratio.

The PC in the figure controls the CCD, the PZT and the XYZ scanner, and processes multiple images with high signalnoise ratio for super-resolution imaging.

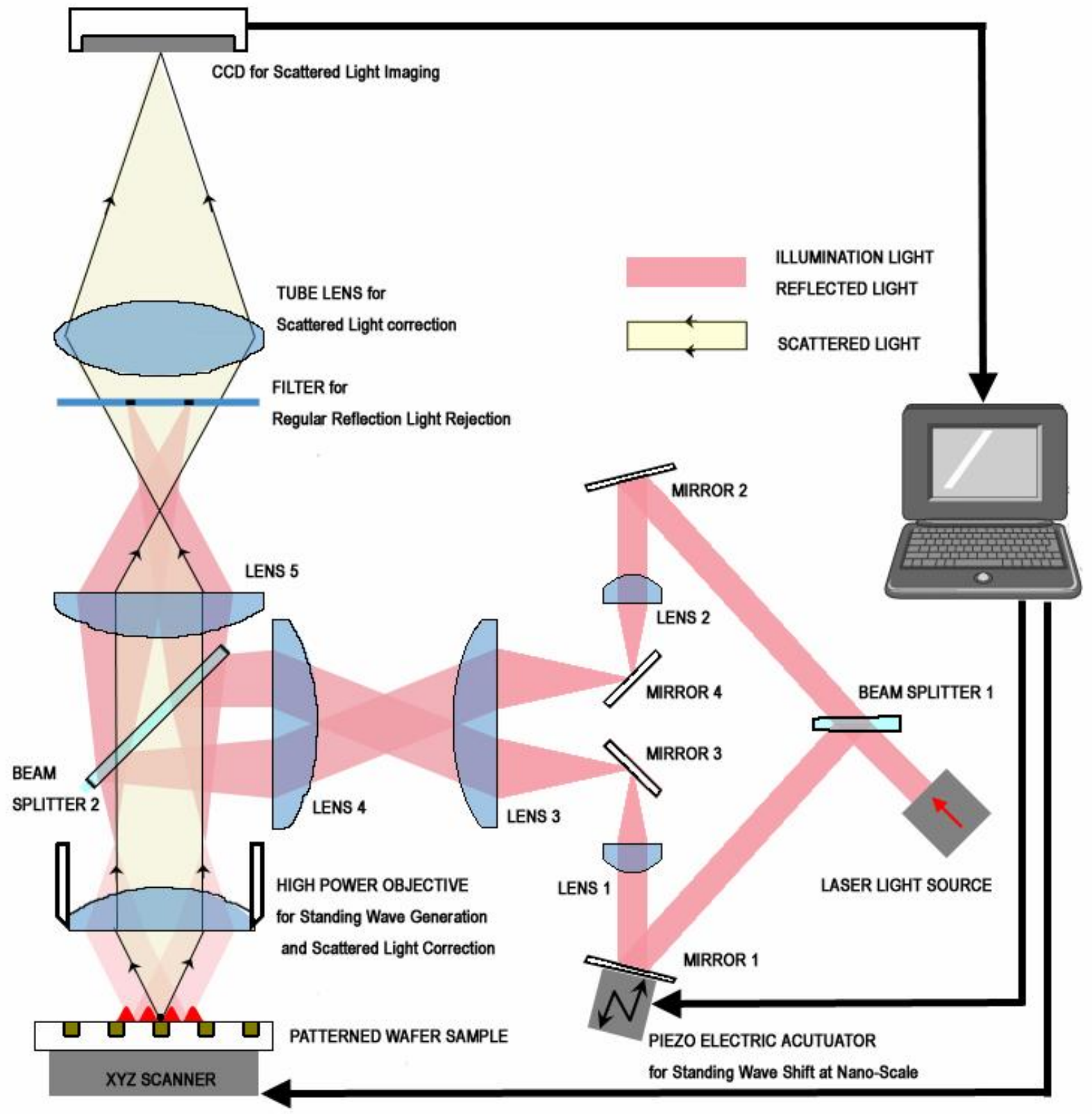

Figure 7. Schematic diagram of the optical system of the super-resolution optical inspection for semiconductor defects using standing wave shift, this optics includes the standing wave illumination generator, the standing wave shift system and the dark-field imaging system using the filter for regular reflection light rejection. 


\section{EXPERIMENT}

For fundamental verification of the super-resolution method, we performed the basic experiment using the developed equipment in the section 3. The experimental setup is shown in table 1. It is expected that the super-resolution method make it possible to observe a structure with higher resolution than conventional resolution depended on NA of an objective lens. And as the verification of the essence of the method, we use the objective lens with relatively low NA (0.46). The super-resolution method assumes the standing wave illumination and the scattered light modulation by the nano-shift of the illumination. The discrete sample is suitable for the scattered light modulation and examination of resolution, so we use the standard particle in the experiment.

Table 1. Experimental setup of the optical system for the super-resolution optical inspection using standing wave illumination shift

\begin{tabular}{|c|c|}
\hline Laser source & Wave length: $488 \mathrm{~nm}$ \\
\hline Piezo electric actuator & Positioning accuracy: $1 \mathrm{~nm}$ \\
\hline Objective lens & NA: $0.46, f=9 \mathrm{~mm}$ \\
\hline Magnification of imaging optics & 29 \\
\hline Filter for regular reflection light rejection & Light interception spot size: $0.5 \mathrm{~mm} \times 2$ \\
\hline CCD camera & Pixel size $8.3 \mu \mathrm{m}, 772 \times 580,16 \mathrm{bit}$ tone \\
\hline Sample & Wafer with $200 \mathrm{~nm}$-sized standard particle \\
\hline
\end{tabular}

\subsection{Confirmation of standing wave illumination and scattered light modulation}

As the preliminary step toward the scattered light imaging, we confirmed generation of the standing wave illumination. The sample with the mirror plane pattern on the wafer is shown in figure 8(a). The sample image is observed with an optical microscope of which magnification is 20 . The sample is illuminated by the standing wave with the developed equipment. In figure $8(\mathrm{~b})$, we show the image of the mirror plane detected with the CCD without the filter for regular reflection light rejection. We confirmed the pitch (peak-to-peak intensity) of the standing wave illumination, which is about $1200 \mathrm{~nm}$. The $3 \mathrm{D}$ magnified view of the standing wave illumination is shown in figure $8(\mathrm{c})$. It was found that the standing wave illumination had the intensity distribution like sine wave. The pitch approximately conformed to the theoretical pitch of the standing wave calculated with the angle between 2-beam.

Next the sample with the standard particles was illuminated by the standing wave through the illumination optics, and the scattered light from the sample was detected with the CCD camera through the dark-field imaging optics. In the figure 9, we plot the scattered light intensity of the peak position of the particle image against the standing wave shift times, in which shift time is corresponding to about $60 \mathrm{~nm}$ lateral shift of the standing wave illumination. We confirmed the linearity of the scattered light intensity against the scattering factor of the particle because the modulation of the scattered light intensity was related the periodic distribution of the standing wave illumination, so we can expect the super-resolution imaging by post-processing of multiple images with an information of the scattered light modulation.

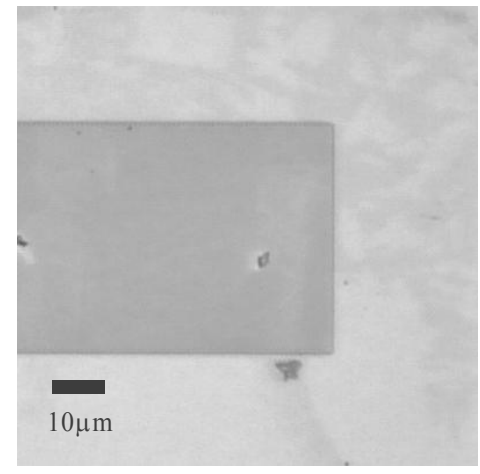

(a)

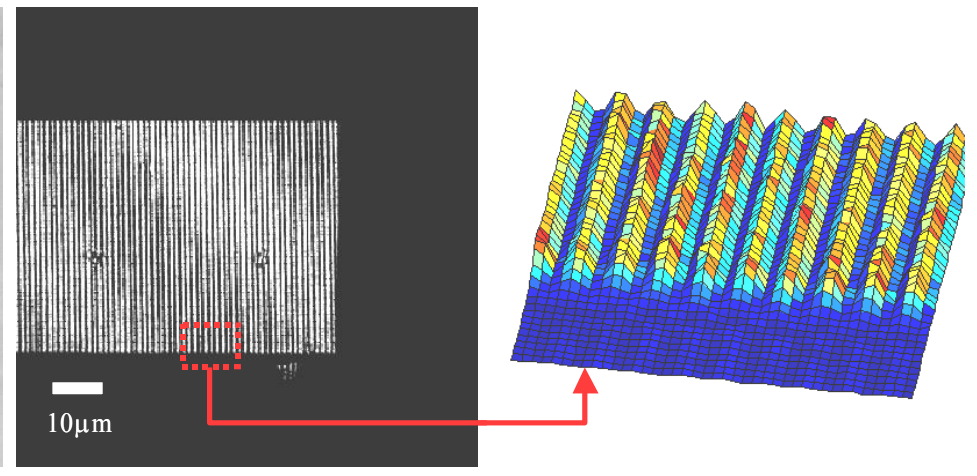

(b) (c)

Figure 8. (a): The sample with the mirror plane pattern on the wafer observed with an optical microscope (20X). (b): The image detected with the developed equipment in the standing wave illumination. (c): The 3D magnified view of the standing wave illumination. 


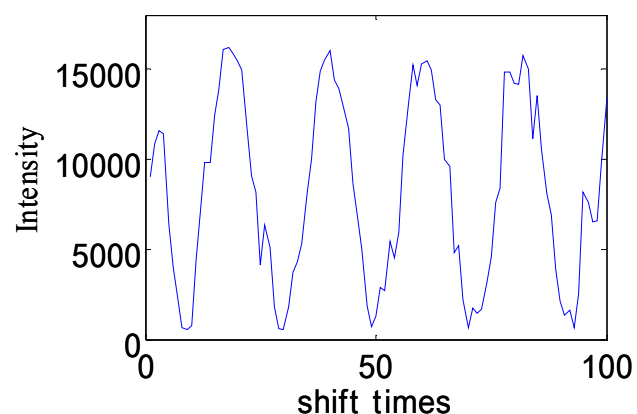

Figure 9. The scattered light intensity of the peak position of the particle image against the standing wave shift times, which 1 time shift distance is about $60 \mathrm{~nm}$.

\subsection{Experiment for super-resolution}

In order to examine the resolution characteristics of the proposed method, we prepared the sample with the 1 particle structure, 2 particles parallel structure and 3 particles parallel structure. The sample with $200 \mathrm{~nm}$-sized particles is shown with a scale in figure 10(a). And the figure 10(b) shows the image detected with the CCD camera in conventional scattered light imaging through the developed optical system (NA: 0.46). In this imaging, relatively lower NA objective lens was used, so it was found that the image of the $200 \mathrm{~nm}$-sized standard particle is not clearly resolved with the conventional method.

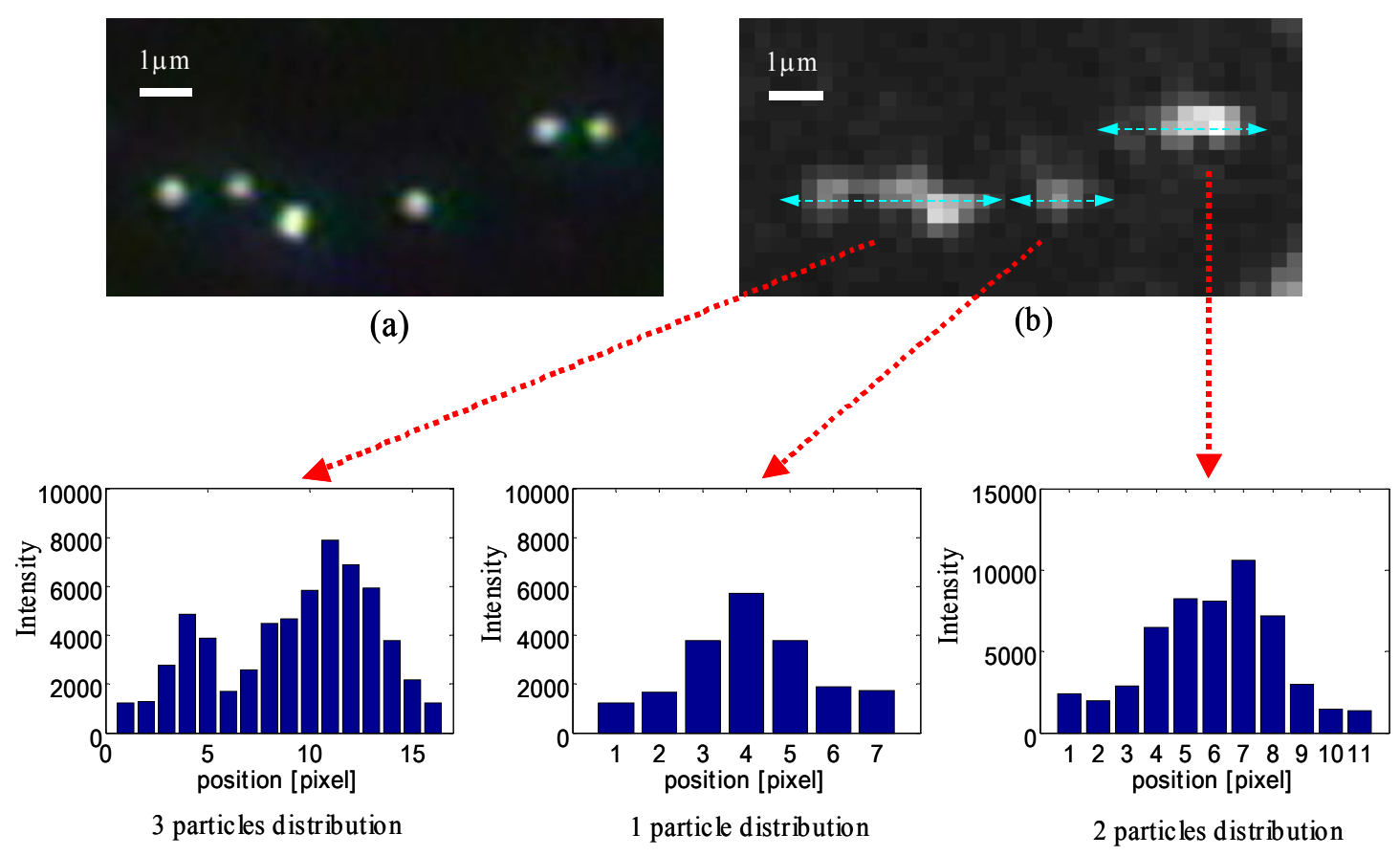

Figure 10. The sample with the 1 particle structure, 2 particles parallel structure and 3 particles parallel structure and the 1D intensity distribution of the scattered light image from 200nm-sized particles, (a): The sample observed with the optical microscope (NA: 0.8). (b): The image detected with the CCD in conventional scattered light imaging through the developed optical system (NA: 0.46).

The super-resolution post-processing was applied for resolving the 200nm-sized standard. The parameters used in the post-processing are shown in table 2 . The prepared sample was illuminated by the standing wave and the illumination was shifted at the step size of $90 \mathrm{~nm}$. We show in the figure 11 the image of the sample modulated by the standing wave illumination shift particles and in the post-processing we used these multiple images with the information of the scattered light modulation. 
The results of the super-resolution post-processing are shown in figure 12, in which (a) shows the post-processed 1D intensity distribution of the image of 1 particle, (b) shows the image of 2 particle and (c) shows the image of 3 particles respectively. We could find in the figure 12 that the $200 \mathrm{~nm}$-sized standard particles were resolved more clearly and had higher contrast compared with the 1D intensity distribution of the particles. Also it was found from the result, the lateral distance of the particles was relatively corresponding to the distance of the particles identified by the optical microscope with high NA (0.8).

Table 2. The parameters used in the super-resolution post-processing

\begin{tabular}{|c|c|}
\hline Illumination light & Wave length: $488 \mathrm{~nm}$ \\
\hline Pitch of standing wave & $1200 \mathrm{~nm}$ \\
\hline Numerical aperture of objective lens & 0.46 \\
\hline Magnification of imaging optics & 29 \\
\hline Standing wave shift step size & $90 \mathrm{~nm}$ \\
\hline Number of images used & 5 \\
\hline Successive approximation times & 2000 \\
\hline
\end{tabular}
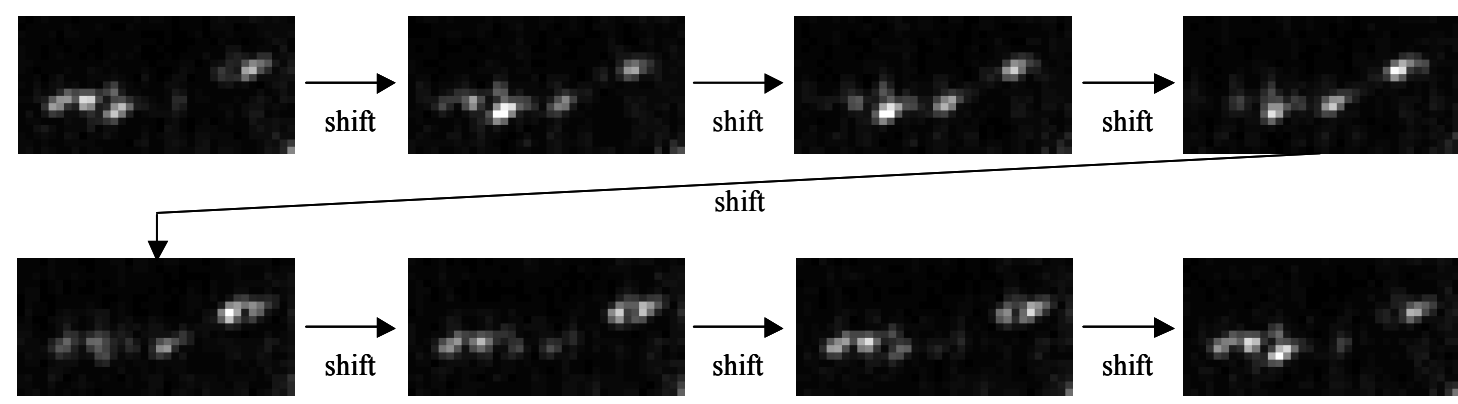

shift

Figure 11. The image of the sample in the standing wave illumination through the developed optics. The image was modulated when the standing wave illumination was shifted.

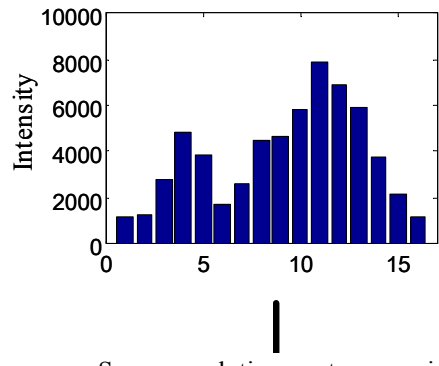

Super-resolution post processing
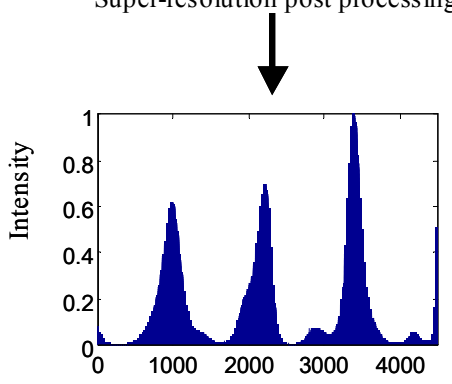

3 partic les distribution

(a)

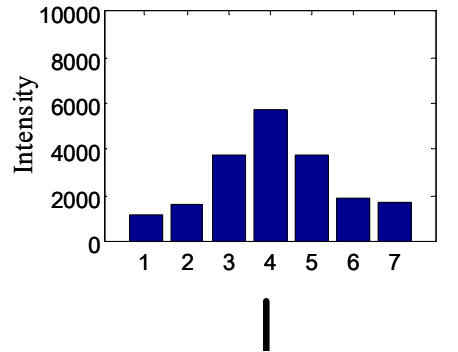

Super-resolution post processing
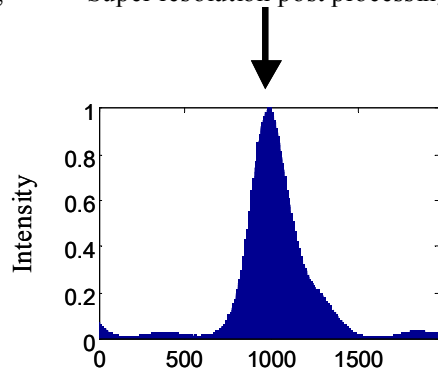

1 particle distribution

(b)

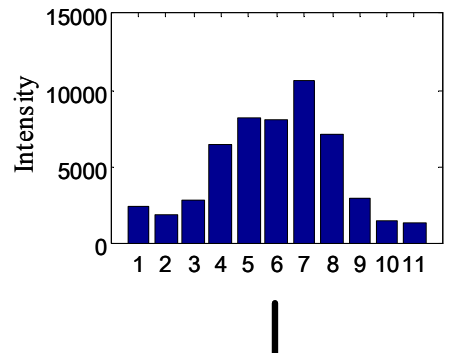

Super-resolution post processing
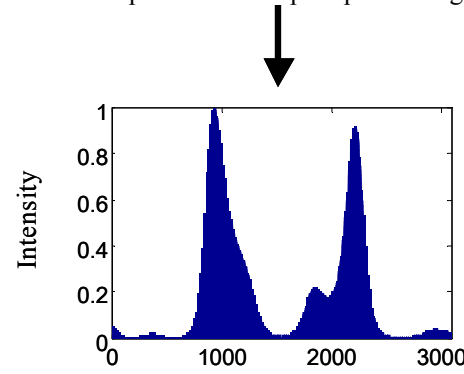

2 particles distribution

(c)

Figure 12. The results of the super-resolution post-processing, in which (a) shows the post-processed 1D intensity distribution of the image of 1 particle, (b) shows the image of 2 particle and (c) shows the image of 3 particles respectively. 


\section{CONCLUSIONS}

In order to verify the super-resolution method fundamentally, we developed the super-resolution optical inspection system using the standing wave illumination. In the performed experiments using developed equipment, the standing wave illumination had the periodic intensity distribution and the scattered light was modulated when the standing wave illumination was shifted, so the scattered light intensity was linear to the scattering factor of the particle. And then, we carried out the basic experiment for super-resolution using the standard particle. As a result, the $200 \mathrm{~nm}$-sized standard particles were resolved more clearly and had higher contrast compared with the conventional microscope image of the particle. And the lateral distance of the particles was relatively corresponding to the distance of the particles. These results suggest that the proposed method make it possible to observe a structure with higher resolution than conventional resolution depended on NA of objective lens. In the future works of the research, we will use high NA objective lens and try to resolve the sub-100nm structure beyond the Rayleigh limit.

\section{REFERENCES}

1. "International Technology Roadmap for Semiconductors, Metrology (2004 update)", Semiconductor Industry Association

2. Mark A. Schulze, Martin A. Hunt, Edgar Voelkl, Joel D. Hickson, William Usry, Randall G. Smith, Robert Bryant and C. E. (Tommy) Thomas Jr. "Semiconductor wafer defect detection using digital holography" Proc. SPIE's Advanced Microelectronic Micromanufacturing, 27-28 February 2003

3. Keisuke Kameyama, Yukio Kosugi, "Semiconductor Defect Classification using Hyperellipsoid Clustering Neural Networks and Model Switching", Proc. IJCNN'99, 1999

4. Jeffery R. Price, Philip R. Bingham, Kenneth W. Tobin, Jr., Thomas P. Karnowski, "Estimating cross-section semiconductor structure by comparing top-down SEM images", Machine Vision Applications in Industrial Inspection XI (Proc. SPIE/IS\&T Vol. 5011), pp.161-170, 2003

5. George W. Mulholland and Thomas A. Germer, "MODELING, MEASUREMENT, AND STANDARDS FOR WAFER INSPECTION", Proc. the Government Microcircuits Applications and Critical Technologies (GOMACTech) Conference, March 31 to April 3, 2003

6. Kenji Watanabe, Shunji Maeda, Tomohiro Funakoshi and Yoko Miyazaki, "DUV Optical Wafer Inspection System for 65-nm Technology Node", Hitachi Review Vol. 54, No. 1, pp22-26, 2005

7. Volker Westphal and Stefan W. Hell, "Nanoscale Resolution in the Focal Plane of an Optical Microscope", PHYSICAL REVIEW LETTERS, No.143903, 2005

8. Yuval Garini, Bart J Vermolen and Ian T Young, "From micro to nano: recent advances in high-resolution microscopy", Current Opinion in Biotechnology, No.16:3-12, 2005

9. SHU-WEI HUANG, HONG-YAO MONG AND CHAU-HWANG LEE, "Super-Resolution Bright-Field Optical Microscopy Based on Nanometer Topographic Contrast", MICROSCOPY RESEARCH AND TECHNIQUE 65, pp180-185, 2004 